\title{
Indicadores de produção assistencial do enfermeiro na Atenção Básica em Saúde
}

\author{
Care production indicators of nurses in Primary Health Care \\ Indicadores de producción de atención de enfermería en Atención Básica de Salud
}

Recebido: 25/01/2021 | Revisado: 04/02/2021 | Aceito: 08/02/2021 | Publicado: 16/02/2021

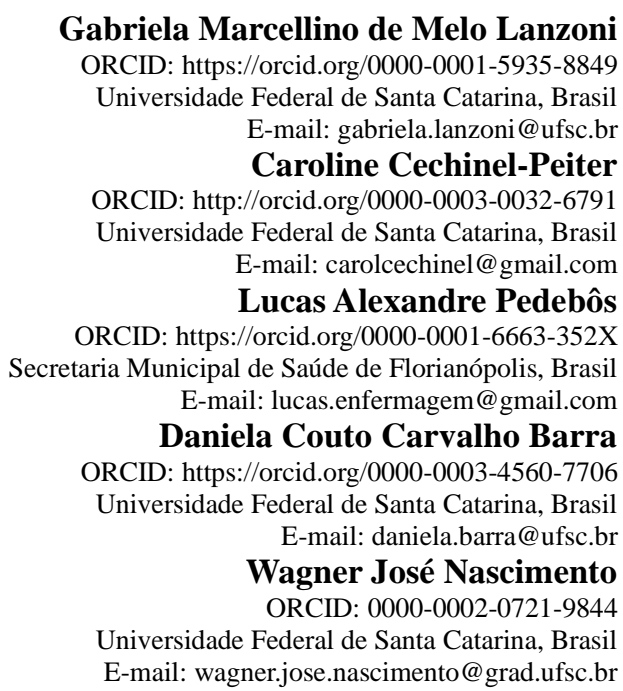

\begin{abstract}
Resumo
Introdução: $\mathrm{O}$ trabalho realizado pelo enfermeiro na Atenção Básica envolve abordagens assistenciais e gerenciais, que podem tornar desafiadora a manutenção da produção assistencial em níveis satisfatórios quando comparada a dos demais profissionais da saúde. Objetivo: Caracterizar os indicadores de produção assistencial de enfermeiros em relação às atividades dos demais profissionais da equipe de saúde da Atenção Básica de Florianópolis, Santa Catarina (SC), Brasil. Método: Estudo exploratório-descritivo e retrospectivo, de abordagem quantitativa. Foi realizada análise em base de dados a partir de registros informatizados da própria instituição relacionados à produção dos trabalhadores das Unidades Básicas de Saúde. Resultados: Enfermeiros realizaram a maioria das visitas domiciliares (56,2\%), e, junto com os médicos, a maioria das consultas $(83,2 \%)$. Dentistas $(33,3 \%)$, psicólogos $(15,1 \%)$ e enfermeiros $(15,0 \%)$ lideraram os atendimentos em grupo, enquanto psicólogos $(25,1 \%)$, assistentes sociais $(19,4 \%)$ e enfermeiros $(19,3 \%)$ participaram com maior frequência de reuniões de equipe. As Unidades Básicas de Saúde com sete equipes tiveram a maior média de consultas de enfermagem por equipe, e a média de procedimentos de enfermagem foi maior naquelas com uma equipe. Conclusão: $\mathrm{O}$ enfermeiro desempenha importante atuação na oferta de atendimentos à população, acesso e resolutividade, mesmo atuando amplamente em atividades de supervisão de equipe.
\end{abstract}

Palavras-chave: Enfermagem; Atenção primária à saúde; Saúde da família; Equipe de enfermagem; Administração de serviços de saúde.

\begin{abstract}
The work performed by nurses in Primary Care involves care and management approaches, which can make it challenging to maintain care production at satisfactory levels when compared to that of other health professionals. Objective: To characterize the indicators of nurses' care production in relation to the activities of other professionals of the Primary Health Care team in Florianópolis, Santa Catarina (SC), Brazil. Method: Exploratory-descriptive and retrospective study, with a quantitative approach. A database analysis was performed based on computerized records of the institution itself related to the production of workers in the Basic Health Units. Results: Nurses performed most home visits $(56.2 \%)$, and, together with doctors, the most consultations (83.2\%). Dentists (33.3\%), psychologists $(15.1 \%)$ and nurses (15.0\%) led group care, while psychologists (25.1\%), social workers (19.4\%) and nurses (19,3\%) participated more frequently in team meetings. It was observed higher mean of nursing consultations in Basic Health Units with seven health teams, and higher mean of technical procedures in Basic Health Units with one health team. Conclusion: Nurses play an important role in providing care to the population, access and resolution, even though they work extensively in team supervision activities.
\end{abstract}

Keywords: Nursing; Primary health care; Family health; Nursing team; Health services administration. 


\section{Resumen}

El trabajo realizado por las enfermeras en Atención Primaria implica enfoques de atención y gestión, lo que puede dificultar el mantenimiento de la producción de atención en niveles satisfactorios en comparación con la de otros profesionales de la salud. Objetivo: caracterizar los indicadores de producción de atención de enfermería en relación con las actividades de otros profesionales del equipo de Atención Primaria de Salud en Florianópolis, Santa Catarina (SC), Brasil Método: estudio exploratorio-descriptivo y retrospectivo, con enfoque cuantitativo. Se realizó un análisis de base de datos basado en registros computarizados de la propia institución relacionados con la producción de trabajadores en las Unidades Básicas de Salud. Resultados: Las enfermeras realizaron la mayoría de las visitas domiciliarias $(56,2 \%)$ y, junto con los médicos, la mayoría de las consultas $(83,2 \%)$. Los dentistas $(33,3 \%)$, los psicólogos $(15,1 \%)$ y las enfermeras $(15,0 \%)$ dirigieron la atención grupal, mientras que los psicólogos $(25,1 \%)$, los trabajadores sociales $(19,4 \%)$ y las enfermeras $(19,3 \%)$ participaron con mayor frecuencia en reuniones de equipo. En las Unidades Básicas de Salud con siete equipos de salud se observó mayor media de consultas de enfermería, y aquellas con un equipo presentó mayor producción en procedimientos técnicos. Conclusión: las enfermeras desempeñan un papel importante en la atención de la población, el acceso y la resolución, a pesar de que trabajan ampliamente en las actividades de supervisión del equipo.

Palabras clave: Enfermería; Atención primaria de salud; Salud familiar; Equipo de enfermería; Administración de servicios de salud.

\section{Introdução}

No Brasil, o Sistema Único de Saúde (SUS) está estruturado em Redes de Atenção à Saúde (RAS), que têm por objetivo a integração de ações e serviços de saúde para prover uma atenção à saúde de forma contínua, integral, de qualidade, responsável e humanizada. Dentre as suas principais características, destaca-se a centralidade da Atenção Básica (AB) na comunicação entre os demais pontos da rede, identificando riscos e demandas de saúde da população por estar cotidianamente mais próxima ao território onde vivem. Desta forma, a AB é considerada ordenadora das redes e coordenadora do cuidado, contribuindo para que a programação dos serviços de saúde parta das necessidades de saúde dos usuários (Rodrigues et al., 2014).

Nesse contexto, a Estratégia Saúde da Família (ESF) visa à reestruturação da AB no país, constituindo-se por equipes para a realização das ações de prevenção de doenças e agravos, promoção e recuperação da saúde de indivíduos e comunidades de forma integral e contínua. Cada equipe é composta minimamente por enfermeiro, médico, técnico ou auxiliar de enfermagem e Agente Comunitário de Saúde (ACS), podendo contar, ainda, com cirurgião dentista e auxiliar de consultório dentário (Vendruscolo, Trindade, Rodrigues, Adamy \& Brum, 2016).

Historicamente, enfermeiros vêm cumprindo importante papel clínico junto à comunidade. No Brasil, há registros da atuação de enfermeiros visitadores a partir de 1916, desempenhando ações de impacto na educação, higiene e administração dos serviços (Campos, Cohn \& Brandão, 2016). A consolidação da ESF possibilitou a ampliação da atuação do enfermeiro no campo comunitário e social (Backes, Backes, Erdmann, Büscher \& Salazar-Maya, 2015).

A força de trabalho da enfermagem brasileira é composta por enfermeiros, técnicos e auxiliares de enfermagem. Dos 2.321.404 profissionais que possuem inscrição ativa no Conselho Federal de Enfermagem, 569.134 (24,5\%) são enfermeiros distribuídos nos diversos campos de atuação (COFEN, 2018).

Considerando que o trabalho realizado pelo enfermeiro na $\mathrm{AB}$ envolve as abordagens assistenciais e gerenciais, entende-se que pode haver dificuldade em manter-se uma produção satisfatória dos atendimentos clínicos no que se refere à qualidade e quantidade, ao mesmo tempo em que se concilia a qualidade das atividades administrativas e gerenciais (Lanzoni, Meirelles \& Cummings, 2016). Assim, observa-se a necessidade de reflexão sobre as lacunas entre o perfil de competências do enfermeiro e as demandas da $\mathrm{AB}$, partindo-se do entendimento que a adequação entre as capacidades dos recursos humanos para responder às necessidades de atenção pode contribuir para o desenvolvimento, fortalecimento e qualificação dos sistemas de saúde.

Além das atividades de gerenciamento da equipe de enfermagem, a supervisão dos ACS, apesar de ser 
responsabilidade de toda a equipe de saúde da $\mathrm{AB}$, ainda é realizada por enfermeiros na maioria dos serviços de saúde (Vendrusculo et al., 2016), o que pode impactar na produção assistencial. Nesse sentido, muitas vezes o pleno potencial do enfermeiro não é reconhecido e acaba sendo subaproveitado. Ainda, a incipiência de estudos sobre os indicadores de produção assistencial do enfermeiro na $\mathrm{AB}$ não permite vislumbrar com clareza o desempenho ideal, impactando globalmente os resultados dos serviços de saúde (Ricciardi, 2018; Smolowitz et al., 2015).

Apesar de estudos internacionais apontarem o potencial de resolutividade e ampliação do acesso oportunizado pela atuação de enfermeiros na AB (Smolowitz et al., 2015; Swan, Ferguson, Chang, Larson \& Smaldone, 2015), estudo realizado na Região Sudeste do Brasil aponta que os atendimentos realizados pelos enfermeiros representaram entre 9,5 e 14,6\% do total, indicando a necessidade de aumento do número de profissionais e da ampliação das ações de enfermagem para oportunizar a resolubilidade esperada para este ponto de atenção (Matumoto et al., 2012). Frente a este contexto, questiona-se: qual a produção do trabalho assistencial do enfermeiro na $\mathrm{AB}$ em um Município de grande porte no Sul do país?

Desse modo, o objetivo deste estudo foi caracterizar os indicadores de produção assistencial de enfermeiros em relação às atividades dos demais profissionais da equipe de saúde da Atenção Básica em um Município de grande porte no Sul do país.

\section{Metodologia}

Trata-se de estudo exploratório, descritivo e retrospectivo, de abordagem quantitativa, caracterizado por observar e descrever fenômenos (Polit \& Beck, 2011), realizado em um município de grande porte no Sul do Brasil. A rede de AB do Município é composta por 49 nas Unidades Básicas de Saúde (UBSs), com cobertura total da ESF, as quais no período de coleta de dados eram distribuídas em cinco distritos sanitários: Centro, Continente, Leste, Norte e Sul. Florianópolis é a capital do Estado de Santa Catarina, possui 485,8 mil habitantes.

Os dados são referentes ao período de janeiro a dezembro de 2016 e foram extraídos do sistema de prontuário eletrônico do município, especificamente, do relatório do desempenho individual dos profissionais de nível superior por UBS (enfermeiro, médico, dentista, fisioterapeuta, nutricionista, farmacêutico, assistente social e psicólogo). Os indicadores analisados foram o número de consultas, visitas domiciliares, atividades na comunidade, grupos terapêuticos e atividades administrativas (reuniões de equipes, reuniões com ACSs). Adicionalmente, como o enfermeiro supervisiona a equipe de enfermagem, buscou-se identificar a produção desenvolvida pelos técnicos de enfermagem por UBS, caracterizada pelo número de procedimentos, destacando-se: curativos, administração de medicações, vacinas, entre outros. Destaca-se que a base de dados do sistema de prontuário utilizado no município é alimentada a partir de registros realizados nos atendimentos dos profissionais.

Das 49 UBSs, três apresentaram dados incompletos no sistema no momento da coleta de dados, sendo excluídas da amostra. Desse modo, foram analisados os dados referentes a 46 UBSs do município. Os dados foram tabulados em planilha do Excel® e posteriormente analisados por meio de estatística descritiva.

Para atender aos princípios éticos, foram observadas e seguidas as recomendações da Resolução no 466/2012 do Conselho Nacional de Saúde (CNS) considerando o respeito pela dignidade humana e pela proteção devida aos participantes de pesquisas científicas envolvendo seres humanos. No desenvolvimento da pesquisa foram cumpridas as solicitações éticas de autorização para o estudo, como a solicitação de autorização à Secretaria Municipal de Saúde de Florianópolis, bem como, a anuência do Comitê de Ética em Pesquisa com Seres Humanos (CEPSH) registrado sob CAAE: 63078916.3.0000.0121.. 


\section{Resultados}

Os dados evidenciaram importante atuação clínica dos enfermeiros, respondendo por 28,7\% das consultas clínicas e mais da metade das visitas domiciliares realizadas no município. Em relação aos demais profissionais, ressalta-se a atuação do farmacêutico que realiza o registro do seu atendimento na dispensação dos medicamentos frente ao paciente, uma vez que esta abordagem é caracterizada como atendimento equivalente à consulta para os demais profissionais de nível superior. Em relação às visitas domiciliares, os enfermeiros lideram a atividade (56,2\%), seguidos pelos médicos $(34,5 \%)$ e dentistas $(15,1 \%)$ (Tabela 1).

Tabela 1 - Produção dos profissionais de ensino superior nas Unidades Básicas de Saúde de Florianópolis. Florianópolis, 2020

\begin{tabular}{cccccc}
\hline Profissional & Número de & \multicolumn{2}{c}{ Consultas } & \multicolumn{2}{c}{ Visitas Domiciliares } \\
& profissionais & N & \% & N & \% \\
\hline Enfermeiro & 224 & 245.747 & 28,7 & 2.926 & 56,2 \\
Médico & 394 & 465.225 & 54,5 & 1.801 & 34,5 \\
Dentista & 92 & 67.906 & 8,0 & 161 & 3,1 \\
Fisioterapeuta & 84 & 7.487 & 0,9 & 128 & 2,5 \\
Nutricionista & 71 & 5.227 & 0,6 & 39 & 0,8 \\
Farmacêutico & 67 & 49.800 & 5,8 & - & - \\
Assistente Social & 67 & 3.160 & 0,4 & 116 & 2,2 \\
Psicólogo & 56 & 9.077 & 1,1 & 37 & 0,7 \\
Total & $\mathbf{1 . 0 5 5}$ & $\mathbf{8 5 3 . 6 2 9}$ & $\mathbf{1 0 0 , 0}$ & $\mathbf{5 . 2 0 8}$ & $\mathbf{1 0 0 , 0}$ \\
\hline
\end{tabular}

Fonte: dados da pesquisa.

Na atenção à saúde da mulher, destaca-se que, do número total de exames preventivos de câncer cérvico-uterino realizados no período, $16.095(99,7 \%)$ foram realizados pelo enfermeiro, enquanto $45(0,3 \%)$ pelo médico, não havendo registro pelos demais profissionais.

Em relação aos atendimentos em grupo, destaca-se a atuação do dentista (33,3\%), psicólogo (15,1\%) e enfermeiro $(15,0 \%)$. Quanto ao número de reuniões de equipe e demais atividades administrativas, houve ênfase da participação de psicólogos $(25,1 \%)$, assistentes sociais $(19,4 \%)$ e enfermeiros $(19,3 \%)$ (Tabela 2$)$. 
Tabela 2 - Atendimentos em grupo e reuniões dos profissionais de ensino superior nas Unidades Básicas de Saúde. Florianópolis, 2020.

\begin{tabular}{ccccc}
\hline & \multicolumn{2}{c}{ Atendimentos em } & \multicolumn{2}{c}{ Reuniões } \\
Profissional & \multicolumn{2}{c}{ grupo } & N & \% \\
\hline Enfermeiro & 1.135 & 15,0 & 797 & 19,3 \\
Médico & 695 & 9,2 & 522 & 12,7 \\
Dentista & 2.515 & 33,3 & 127 & 3,1 \\
Fisioterapeuta & 948 & 12,6 & 264 & 6,4 \\
Nutricionista & 586 & 7,8 & 182 & 4,4 \\
Farmacêutico & 84 & 1,1 & 395 & 9,6 \\
Assistente Social & 448 & 5,9 & 799 & 19,4 \\
Psicólogo & 1.143 & 15,1 & 1.038 & 25,1 \\
Total & $\mathbf{7 . 5 5 4}$ & $\mathbf{1 0 0 , 0}$ & $\mathbf{4 . 1 2 4}$ & $\mathbf{1 0 0 , 0}$ \\
\hline
\end{tabular}

Fonte: dados da pesquisa.

Os indicadores de produção assistencial da equipe de enfermagem foram analisados em relação ao número de equipes das UBSs. As UBSs com sete equipes tiveram a maior média de consultas de enfermagem por equipe, enquanto a média de procedimentos de enfermagem foi maior em UBSs com uma equipe, sendo as UBSs com até quatro equipes as mais produtivas sob esta ótica (Tabela 3). 
Tabela 3 - Consultas de enfermagem e procedimentos realizados pela equipe de enfermagem nas Unidades Básicas de Saúde com base no número de equipes. Florianópolis, 2020.

\begin{tabular}{|c|c|c|c|c|c|c|c|c|c|}
\hline \multirow{2}{*}{$\begin{array}{c}\text { Equipes } \\
\mathbf{N}\end{array}$} & \multirow{2}{*}{$\begin{array}{c}\text { Unidades } \\
\mathbf{N}(\%)\end{array}$} & \multicolumn{4}{|c|}{ Consultas de enfermagem } & \multicolumn{4}{|c|}{ Procedimentos de enfermagem } \\
\hline & & $\mathbf{N}(\%)$ & $\begin{array}{c}\text { Média } \\
\text { por } \\
\text { equipe }\end{array}$ & $\begin{array}{c}\text { Mediana } \\
\text { por } \\
\text { UBS* }\end{array}$ & $\mathbf{D} \mathbf{P}^{\dagger}$ & $\mathbf{N}(\%)$ & $\begin{array}{c}\text { Média } \\
\text { por } \\
\text { equipe }\end{array}$ & $\begin{array}{c}\text { Median } \\
\text { a por } \\
\text { UBS* }\end{array}$ & $\mathbf{D} \mathbf{P}^{\dagger}$ \\
\hline 8 & $1(2,2)$ & $13.430(5,5)$ & 1.679 & 13.430 & - & $\begin{array}{c}18.413 \\
(4,5)\end{array}$ & 2.302 & 18.413 & - \\
\hline 7 & $1(2,2)$ & $15.001(6,1)$ & 2.143 & 15.001 & - & $\begin{array}{c}15.711 \\
(3,8)\end{array}$ & 2.244 & 15.711 & - \\
\hline 6 & $2(4,3)$ & $\begin{array}{l}24.694 \\
(10,0)\end{array}$ & 2.058 & 12.347 & 6.531 & $\begin{array}{c}27.224 \\
(6,6)\end{array}$ & 2.269 & 13.612 & 1.295 \\
\hline 5 & $4(8,7)$ & $\begin{array}{l}27.151 \\
(11,0)\end{array}$ & 1.358 & 6.998 & 2.454 & $\begin{array}{c}43.944 \\
(10,6)\end{array}$ & 2.197 & 11.588 & 2.824 \\
\hline 4 & $6(13,0)$ & $\begin{array}{c}46.716 \\
(19,1)\end{array}$ & 1.947 & 8.541 & 3.816 & $\begin{array}{l}76.333 \\
(18,4)\end{array}$ & 3.181 & 13.124 & 3.138 \\
\hline 3 & $11(24,0)$ & $\begin{array}{l}61.225 \\
(24,9)\end{array}$ & 1.855 & 5.348 & 2.029 & $\begin{array}{c}103.394 \\
(25,0)\end{array}$ & 3.133 & 8.342 & 2.585 \\
\hline 2 & $14(30,4)$ & $\begin{array}{c}47.654 \\
(19,4)\end{array}$ & 1702 & 3.256 & 1.753 & $\begin{array}{l}99.582 \\
(24,1)\end{array}$ & 3.557 & 6.667 & 3.781 \\
\hline 1 & $7(15,2)$ & $9.876(4,0)$ & 1.411 & 1.240 & 851 & $\begin{array}{c}29.075 \\
(7,0)\end{array}$ & 4.154 & 3.331 & 2.512 \\
\hline Total & $46(100,0)$ & $\begin{array}{r}245.747 \\
(100,0)\end{array}$ & 1.755 & 4.623 & 3.777 & $\begin{array}{c}413.676 \\
(100,0)\end{array}$ & 2.955 & 8.040 & 4.374 \\
\hline
\end{tabular}

*UBS - Unidade Básica de Saúde; †DP - Desvio Padrão. Fonte: dados da pesquisa.

\section{Discussão}

Conforme orientam a Política Nacional de Atenção Básica (PNAB) e a Política Nacional de Humanização, o acesso aos serviços de saúde nas UBSs de Florianópolis ocorre de forma programática e em livre demanda por meio do acolhimento (Vendrusculo et al., 2016). Embora o acolhimento dos usuários não seja responsabilidade exclusiva de enfermeiros e médicos, estes são os profissionais que frequentemente assumem esta atividade, respondendo pelo primeiro contato com o usuário (Santos et al., 2016), fato este que pode ter contribuído para o alto número de consultas registradas por estes profissionais no presente estudo.

Apesar da consulta programática ser culturalmente atribuição do médico, o enfermeiro vem ganhando espaço nesta função e seu protagonismo na ESF muito tem contribuído para este avanço (Campos et al., 2016). Estudo realizado em dez estados no Brasil verificou que em municípios de grande porte, como no caso da realidade investigada, é mais frequente a atuação do enfermeiro em ações individuais de cuidado clínico como a consulta e acolhimento, ao mesmo tempo que este profissional despende um percentual inferior de tempo a atividades educativas e ações coletivas, ao contrário do que acontece em municípios de pequeno porte (Bonfim, Fugulin, Laus, Peduzzi \& Gaidzinski, 2016).

Prática privativa do enfermeiro, a consulta de enfermagem inclui avaliação das necessidades de saúde, sinais de gravidade, diagnóstico de enfermagem, e condução adequada do caso, incluindo tratamento quando necessário e validado por 
protocolos. Além disso, permite a realização de atividades de educação em saúde e a consolidação do vínculo entre profissional e paciente, promovendo relações de confiança. Trata-se, portanto, de uma atividade substancial à estrutura da $\mathrm{AB}$, uma vez que enfoca ações de autocuidado e orientação do paciente para suas necessidades (Vendrusculo et al., 2016; Santos et al., 2016).

Em relação à atuação do farmacêutico, sua interação com o usuário durante a dispensação de medicamentos na realidade investigada é registrada como consulta, uma vez que é equivalente ao atendimento realizado pelos demais profissionais de nível superior. No contexto do SUS, o objetivo da dispensação de medicamentos vai além da abordagem limitada ao fornecimento do insumo, sendo um importante momento para o profissional se aproximar das necessidades do usuário e população (Leite et al., 2017) e facilitar a adesão ao tratamento proposto, ou mesmo realizar ajustes nesse, diante das possibilidades legais da profissão.

Para a obtenção de um conhecimento ainda mais amplo acerca do indivíduo e do meio no qual o mesmo se encontra inserido, a ESF prevê a cobertura populacional através de áreas geográficas, cuja delimitação deve considerar a diversidade sociopolítica, econômica, densidade populacional, acessibilidade aos serviços, entre outros fatores, definindo, assim, o território de atuação das equipes. Dentre as atribuições da equipe de saúde da família, a visita domiciliar (VD) destaca-se como um instrumento capaz de promover a inserção da equipe no território e o conhecimento da realidade vivida pela comunidade e, consequentemente, o estabelecimento de vínculos entre profissionais e indivíduos, permitindo uma maior proximidade acerca dos determinantes do processo saúde-doença (Kebian \& Acioli, 2014).

Apesar de ser atribuição de todos os profissionais da AB (Vendrusculo et al., 2016), os dados deste estudo indicaram que as VDs são realizadas majoritariamente pelo enfermeiro e em menor proporção pelo médico de família e clínico geral, com discreta participação dos demais profissionais da equipe multidisciplinar. O incentivo à participação da equipe de saúde nas VDs se deve por esta proporcionar um olhar mais abrangente sobre os determinantes do processo saúde-doença, e auxiliar diretamente no planejamento terapêutico e nas intervenções da equipe multiprofissional em saúde, fornecendo assim, a excelência no cuidado integral, embasado nos conhecimentos técnico-científicos e na realidade vivenciada pela comunidade (Santos et al., 2016; Kebian \& Acioli, 2014).

Estudo realizado na Inglaterra apontou que, para a gestão de casos clínicos acontecer de maneira efetiva, é indispensável a atuação conjunta do enfermeiro com os demais membros da equipe, permitindo, por exemplo, a manutenção de pacientes crônicos em cuidado domiciliar e evitar, assim, sua reospitalização e oneração indevida do sistema de saúde (Soares \& Silva, 2016).

No que se refere à resolutividade do profissional enfermeiro, a área da saúde da mulher tem importante destaque com o desenvolvimento de orientações e procedimentos, como o exame preventivo de câncer cérvico-uterino (Souza \& Costa, 2015). A realização do exame é prática comumente atribuída ao enfermeiro, que deve valer-se desse momento para ampliar sua atuação, incorporando outras práticas preventivas, de promoção e educação em saúde (Nicolau, Espírito Santo, David, 2017). Para a consolidação de uma prática segura e resolutiva, os protocolos clínicos são importantes ferramentas no processo de cuidar. No contexto em estudo, a partir de 2015 protocolos clínicos foram elaborados e implantados após capacitação para todos os enfermeiros da rede.

As atividades de cunho coletivo, seja com pacientes ou com os próprios profissionais, através de reuniões, visam responder as demandas plurivalentes advindas dos diferentes segmentos, sejam crianças, mulheres, idosos ou adultos em situações crônicas ou agudas. No desenvolvimento de grupos terapêuticos, teve destaque o dentista, fato que se dá pelas frequentes atividades realizadas em escolas e por grupos de acolhimento a recém-nascidos.

Embora não tenha sido objetivo do estudo investigar se houve participação de mais de um profissional em cada grupo terapêutico, vislumbra-se que a atuação interdisciplinar potencializa o processo de orientação aos usuários e famílias no 
contexto da $\mathrm{AB}$ em diversas situações saúde-doença, como na obesidade (Nicolau et al., 2017), na saúde mental (Slade et al., 2015), e na adesão ao tratamento medicamentoso com parceria com farmacêuticos (Celio, Ninane, Bugnon \& Schneides, 2018). Nessa conjuntura, a atuação organizada e integrada dos profissionais de saúde permite uma abordagem mais ampla do indivíduo e, consequentemente, possibilita um cuidado mais efetivo. $\mathrm{O}$ trabalho integrado da equipe multiprofissional viabiliza a implantação de um trabalho cooperativo, com múltiplas intervenções técnicas e interação de profissionais de distintas profissões, permitindo uma abordagem ao indivíduo de forma integral e ampliada (Nicolau et al., 2017).

Embora sejam reconhecidas as dificuldades no atendimento aos indivíduos que recorrem às UBSs, a enfermagem atenta-se de forma permanente para com a melhoria da assistência, buscando conhecimentos que possam organizar e sistematizar sua prática de cuidado, favorecendo não somente uma assistência voltada à dimensão biológica do ser humano, mas na percepção do ser humano como um ser social e de seu processo de saúde-doença, seja no contexto hospitalar ou na saúde coletiva.

No que respeito à capacidade de trabalho dos profissionais, é oportuno ressaltar que, devido às habilidades e competências desenvolvidas durante a formação acadêmica do enfermeiro, a produtividade e bom aproveitamento da sua força de trabalho influencia diretamente no acesso e qualidade da atenção integral ao usuário e população, indicando a necessidade de incentivos para o maior engajamento de enfermeiro nas práticas assistenciais da AB (Bonfim et al., 2016; Salinas, Lopez \& Ampuero, 2020; Gallotti at al, 2021). No entanto, no contexto da AB é evidenciado desconexão entre a capacidade clínica do enfermeiro e a complexidade de suas atribuições. $\mathrm{O}$ aumento do escopo de atuação do enfermeiro deve se dar como estratégia de qualificação do sistema de saúde, sob uma lógica colaborativa na qual possa ser priorizada a capacidade coletiva da equipe. Logo, a utilização adequada do enfermeiro vem a contribuir com a assistência prestada por toda a equipe, sem o intuito de substituir outros profissionais, mas complementá-los (Ricciardi, 2018).

Nos Estados Unidos, mediante a formação complementar com diferentes ênfases definidas pela legislação, enfermeiros de práticas avançadas demonstraram resultados iguais ou superiores a médicos, no que se refere à resposta fisiológica, satisfação do paciente e custo para o serviço de saúde (Swan et al., 2015). O cenário investigado se destaca no âmbito nacional pela estrutura da $\mathrm{AB}$, apresentando semelhanças ao movimento que aconteceu no sistema de saúde do Reino Unido, em que o enfermeiro passou a desenvolver competências profissionais ampliadas, incluindo atividades clínicas e nãoclínicas, com ênfase no manejo de condições crônicas. Com isso, por meio de protocolos, os enfermeiros passaram a atuar no tratamento e acompanhamento de pacientes com condições crônicas. Ainda, no Reino Unido, desde 1992, os enfermeiros são habilitados a realizar a prescrição de medicamentos com base em protocolos e formação complementar. Nessa conjuntura, enfermeiros representam $43 \%$ dos profissionais prescritores na atenção primária neste país, o que gerou resultados positivos no que tange a relação custo-efetividade para o sistema de saúde e população (Toso, Filippon \& Giovanella, 2016).

Nesse sentido e tendo em vista a mudança do perfil epidemiológico e a tripla carga de doenças, para que os sistemas de saúde se adequem ao aumento das demandas e mudanças das necessidades das populações, é necessário o investimento na revisão e adaptação dos currículos de enfermagem para que preparem a força de trabalho para assumir papeis ampliados. Essas reformulações sugeridas na formação do enfermeiro exigem enfoque sobre as habilidades, conhecimentos e tomada de decisão necessários no contexto da $\mathrm{AB}$ (Wojnar \& Whelan, 2017).

Na Austrália, o escopo de atuação clínica do enfermeiro vem aumentando consideravelmente desde 2012. A prática do enfermeiro em cada contexto depende de fatores ambientais, políticos e das necessidades da população, sendo comum a sobreposição de atividades e responsabilidades entre os membros da equipe. Além disso, assim como no Brasil, na Austrália a atuação do enfermeiro na $\mathrm{AB}$ vai desde a abordagem a situações agudas a condições crônicas, englobando ações de promoção e educação em saúde. Essa característica acarreta em um desafio para a caracterização e definição do escopo de atuação do enfermeiro na AB (Halcomb, Stephens, Bryce, Foley \& Ashley, 2017). 
Estudo que mapeou a produção científica sobre o trabalho do enfermeiro no Brasil identificou que os estudos analisados apresentaram maior preocupação em detalhar pequenas mudanças no rol de atribuições do profissional, em detrimento de uma discussão mais ampla sobre a reorganização do trabalho do enfermeiro frente às mudanças do modelo de atenção à saúde (Souza, Teixeira \& Souza, 2017). Ou seja, frente à emergência do modelo de tripla carga de doenças, em que prevalecem os agravos crônicos em coocorrência às condições agudas, tem-se a oportunidade de avançar-se no escopo de atuação do enfermeiro com melhor utilização de sua expertise técnica, oportunizando melhoria do acesso, longitudinalidade e qualidade do serviço.

Em coerência com os achados deste estudo, pesquisa que objetivou identificar os padrões das atividades dos profissionais de enfermagem no Brasil verificou que as atividades mais frequentes realizadas pelo enfermeiro na $\mathrm{AB}$ incluem consulta de enfermagem, VD e atendimento à demanda espontânea ou acolhimento. No entanto, 7\% das atividades dos enfermeiros e $14 \%$ daquelas desenvolvidas por técnicos de enfermagem são caracterizadas como atividades que poderiam ser executadas por profissionais de outras categorias (Peiter, Caminha \& Oliveira, 2017), o que diminui a disponibilidade do enfermeiro e equipe para desempenhar atividades assistenciais diretas que fortaleçam a perspectiva assistencial da profissão.

Dentre estas demandas, pode-se considerar as atividades gerenciais, uma vez que a coordenação das UBSs é na maioria dos casos atribuída aos enfermeiros (Nunes, Pires \& Machado, 2020; Reis, Almeida, Andrade \& Daltro, 2016). Quando não atribuídas a um profissional específico para desempenho da função, o acúmulo de atividades leva à diluição do tempo do profissional de dedicação às atividades assistenciais. Nesse sentido, destaca-se a importância de se compreender a organização dos serviços e as práticas profissionais incluídas nesta para a produção efetiva do cuidado (Soder et al., 2020).

Como limitações desse estudo apontam-se o uso exclusivo de dados secundários, oriundo do registro dos profissionais do serviço e a análise de um único município, o que impede a generalização dos resultados.

\section{Conclusão}

A produção assistencial dos enfermeiros na realidade investigada tem destaque em ações diretas como a consulta de enfermagem, VD, assistência à saúde da mulher, e indiretas como reuniões de equipe e atendimentos em grupo. Apesar de o enfermeiro desempenhar importante atuação na oferta de atendimentos à população, os resultados evidenciaram necessidade de maior engajamento dos demais profissionais da equipe nas atividades coletivas que possam se beneficiar da interação multiprofissional. A responsabilização de atividades coletivas pelo enfermeiro, quando não assumidas de modo corresponsável pelos demais profissionais, impede o total aproveitamento de sua habilidade clínica. Nesse sentido, a apropriação de práticas avançadas pelo enfermeiro na $\mathrm{AB}$ permite que os serviços de saúde estejam preparados para a crescente demanda assistencial, contribuindo com a qualificação da equipe e melhoria da assistência prestada.

Espera-se que este estudo incentive novas investigações sobre o aproveitamento assistencial adequado do enfermeiro e seu potencial de contribuição na qualificação do sistema de saúde. Novos estudos são recomendados no sentido de ampliar a caracterização e compreensão da produção assistencial do enfermeiro na Atenção Básica, permitindo a comparação entre diferentes contextos e generalização dos achados.

\section{Financiamento}

O presente trabalho contou com apoio da Coordenação de Aperfeiçoamento de Pessoal de Nível Superior - Brasil (CAPES) - Código de Financiamento 001. 


\section{Referências}

Backes, D. S., Backes, M. S., Erdmann, A. L., Büscher, A. \& Salazar-Maya, A. M. (2015). Significance of the Nurse's Social Practice with and through the Unified Brazilian Health Care System. Aquichan, 14(4), 560-70. https://doi.org/10.5294/aqui.2014.14.4.10

Bonfim, D., Fugulin, F. M. T., Laus, A. M., Peduzzi, M. \& Gaidzinski, R. R. (2016). Time standards of nursing in the Family Health Strategy: an observational study. Rev Esc Enferm USP, 50(1), 121-129. http://dx.doi.org/10.1590/S0080-623420160000100016

Campos, C. E. A., Cohn, A. \& Brandão, A. L. (2016). The historical trajectory of the city of Rio de Janeiro's health system: 1916-2015. One hundred years of innovations and achievements. Ciênc Saúde Colet, 21(5), 1351-64. http://dx.doi.org/10.1590/1413-81232015215.00242016

Celio, J., Ninane, F., Bugnon, O. \& Schneides, M. P. (2018). Pharmacist-nurse collaborations in medication adherence-enhancing interventions: A review. Patient educ couns, 101(7), 1175-1192. https://doi.org/10.1016/j.pec.2018.01.022

Conselho Federal de Enfermagem de Santa Catarina (COFEN) (2018). Enfermagem em números. Disponível em: http://www.cofen.gov.br/enfermagem-emnumeros

Galloti, F. C. M., Santos, L. E. R., Dias, V. G. A., Farias, Q. S. S., Martins, M. C. V., Góis, R. M. O., Lima, R. G., Trindade, L. S., Serafini, M. R. (2021). Formação do enfermeiro na perspectiva do cuidado integral e trabalho em equipe. Research, Society \& Development, 10(1), e224110111724. http://dx.doi.org/10.33448/rsd-v10i1.11724

Halcomb, E., Stephens, M., Bryce, J., Foley, E. \& Ashley, C. (2017). The development of professional practice standards for Australian general practice nurses. J adv nurs, 73(8), 1958-69. https://doi.org/10.1111/jan.13274

Kebian, L. V. A. \& Acioli, S. (2014). Home visits by Family Health Strategy nurses and community health agents. Rev eletrônica enferm, 16(1), 161-9. http://dx.doi.org/10.5216/ree.v16i1.20260

Lanzoni, G. M. M., Meirelles, B. H. S. \& Cummings, G. (2016). Nurse leadership practices in primary health care: a grounded theory. Texto Contexto Enferm, 25(4), e4190015. http://dx.doi.org/10.1590/0104-07072016004190015

Leite, S. N., Bernardo, N. L. M. C., Guerra Junior, A. A., Costa, E. A., Acurcio, F. A., Guibu, I. A., Costa, K. S., Karnikowski, M. G. O., Soeiro, O. M. \& Soares, L. (2017). Medicine dispensing service in primary health care of SUS. Rev saúde pública, 51(Supl 2), 11s. https://doi.org/10.11606/S15188787.2017051007121

Matumoto, S., Vieira, K. C. S., Pereira, M. J. B., Santos, C. B., Fortuna, C. M. \& Mishima, S. M. (2012). Production of nursing care in primary health care services. Rev latinoam enferm, 20(4), 710-7. http://www.scielo.br/pdf/rlae/v20n4/11.pdf

Nicolau, I. R., Espírito Santo, F. H., David, F. M. (2017). Teamwork: in search of qualified care for the obese. Rev enferm UFPE on line, 11(1), 152-9. https://periodicos.ufpe.br/revistas/revistaenfermagem/article/view/11888

Nunes, A. C. B., Pires, D. E. P. \& Machado, R. R. (2020). Satisfação e insatisfação no trabalho de gestores na estratégia saúde da família. Cogitare Enfermagem, 25(1). https://revistas.ufpr.br/cogitare/article/view/61440.

Peiter, C. C., Caminha, M. E. P. \& Oliveira, W. F. (2017). Managers' profile in Primary Health Care: an integrative review. Espaç. saúde (Online), 18(1), 165173. http://espacoparasaude.fpp.edu.br/index.php/espacosaude/article/view/357

Polit, D. F., Beck, C. T. (2011). Fundamentos de pesquisa em enfermagem: avaliação de evidências para a prática de enfermagem. Porto Alegre: Artmed.

Reis, A. P., Almeida, M. F. A., Andrade, I. R. \& Daltro, E. F. M. A. (2016). Institutional support for primary care management: an experience report. Rev baiana saúde pública, 40(2), 532-42. http://rbsp.sesab.ba.gov.br/index.php/rbsp/article/view/2112

Ricciardi, R. (2018). The Next Frontier for Nurses: Improving Quality and Safety in Primary Care. $J$ nurs care qual, 33(1), 1-4. https://www.ncbi.nlm.nih.gov/pubmed/29166303

Rodrigues, L. B. B., Silva, P. C. S., Peruhype, R. C., Palha, P. F., Popolin, M. P., Crispim, J. A., Pinto, I. C., Monroe, A. A. \& Arcêncio, A. A. (2014). Primary Health Care in the coordination of health care networks: an integrative review. Ciênc Saúde Colet, 19(02), 343-51. https://doi.org/10.1590/141381232014192.18032012

Salinas, J. V. R., Lopez, J. J. G. \& Ampuero, E. V.C. (2020). Índice de capacidad de trabajo en enfermeras en atención primaria de salud, Lima-Perú. Avances en Enfermería, 38(1), 37-45. https://doi.org/10.15446/av.enferm.v38n1.79693

Santos, D. A., Shirasaki, R. T. S., Cangussu, J. M. L., Fermino, J. M., Silva, A. T. \& Campos, G. L. (2016). Potential and difficulties in user embracementin primary care network as the National Policy of Humanization. Saúde transform soc, 6(2), 54-69. http://stat.necat.incubadora.ufsc.br/index.php/saudeetransformacao/article/view/3313/4470

Slade, M., Bird, V., Clarke, E., Boutillier, C. L., McCrone, P., McPherson, R., Pesola, F., Wallace, G., Willians, J. \& Leamy, M. (2015). Supporting recovery in patients with psychosis through care by community-based adult mental health teams (REFOCUS): a multisite, cluster, randomised, controlled trial. Lancet Psychiatry, 2, 503-14. https://doi.org/10.1016/S2215-0366(15)00086-3

Smolowitz, J., Speakman, E., Wojnar, D., Whelan, E. M., Ulrich, S., Hayes, C. \& Wood, L. (2015). Role of the registered nurse in primary health care: Meeting health care needs in the 21st century. Nurs Outlook, 62(2), 130-6. https://doi.org/10.1016/j.outlook.2014.08.004

Soares, M. B. O. \& Silva, S. R. (2016). Interventions that facilitate adherence to Pap smear exam: integrative review. Rev bras enferm, 69(2), 381-91. http://dx.doi.org/10.1590/0034-7167.2016690226i 
Research, Society and Development, v. 10, n. 2, e29410212354, 2021

(CC BY 4.0) | ISSN 2525-3409 | DOI: http://dx.doi.org/10.33448/rsd-v10i2.12354

Soder, R. M., Santos, J. L. G., Santos, L. E., Oliveira, I., Silva, L. A. \& Peiter, C. C. (2020). Práticas de enfermeiros na gestão do cuidado na atenção básica. Rev Cubana Enferm, 36(1). http://www.revenfermeria.sld.cu/index.php/enf/article/view/2815

Souza, A. F. \& Costa, L. H. R. (2015). Cervical Cancer and HPV: Women's Knowledge after Nursing Consultation. Rev Bras Cancerol, 61(4), 343-50. $\mathrm{http} / / / \mathrm{www}$.inca.gov.br/Rbc/n_61/v04/pdf/05-artigo-conhecimento-de-mulheres-sobre-hpv-e-cancer-do-colo-do-utero-apos-consulta-de-enfermagem.pdf

Souza, E. A., Teixeira, C. F. \& Souza, M. K. B. (2017). Analysis of the national scientific production on the work of nurses (1988-2014). Saúde debate, 41(113), 630-646. http://dx.doi.org/10.1590/0103-1104201711322

Swan, M., Ferguson, S., Chang, A., Larson, E. \& Smaldone, A. (2015). Quality of primary care by advanced practice nurses: a systematic review. Int $j$ qual health care, 27(5), 396-404. https://doi.org/10.1093/intqhc/mzv054

Toso, B. R. G. O., Filippon, J. \& Giovanella, L. (2016). Nurses' performance on primary care in the National Health Service in England. Rev bras enferm, 69(1), 182-91. http://dx.doi.org/10.1590/0034-7167.2016690124i

Vendruscolo, C., Trindade, L. L., Rodrigues, O. C. C., Adamy, E. K. \& Brum, M. L. B. (2016). Introductory to family health teams: contributions to the strengthening of primary care. Rev enferm UFPE on line, 10(9), 3393-400. https://periodicos.ufpe.br/revistas/revistaenfermagem/article/view/11421

Wojnar, D. M. \& Whelan, E. M. (2017). Preparing nursing students for enhanced roles in primary care: The current state of prelicensure and RN-to-BSN education. Nurs Outlook, 65(2), 222-232. https://doi.org/10.1016/j.outlook.2016.10.006 